Manuelle Medizin 2020 · 58:95-96

https://doi.org/10.1007/s00337-020-00691-5

(c) Springer Medizin Verlag GmbH, ein Teil von Springer Nature 2020

Im Jahr 2019 hatten Vinzelberg et al. in einem Beitrag „Systematisierung und Standardisierung manueller Untersuchungsund Behandlungstechniken" darauf aufmerksam gemacht, dass in vielen Publikationen „der Einsatz manueller Interventionen überwiegend nur allgemein und pauschal als ,manualmedizinisch behandelt' oder ,osteopathisch behandelt beschrieben wird, ohne die präzise Ausführung der angewandten Techniken genauer anzugeben " [1]. Die Autoren untermauerten ihre Forderung nach genauer Beschreibung sowohl des Vorgehens bei der Untersuchung als auch bei den therapeutischen Techniken, indem sie sich u. a. auf Alvares et al. [2] beriefen, welche eine Leitvorlage für die Beschreibung therapeutischen Vorgehens und deren Nachvollziehbarkeit forderten und publizierten. Ein Anlass, in der Zeitschrift Manuelle Medizin auf die Notwendigkeit einer genaueren Beschreibung manualmedizinischer Vorgehensweisen in den genannten Beiträgen hinzuweisen, waren auch die ungenügend oder ungenau beschriebenen Prozeduren der Intervention in den zur Publikation in Manuelle Medizin (MM) eingereichten Manuskripten.

Offensichtlich nehmen einige $\mathrm{Au}-$ toren an, dass der Hinweis „es wurde manualmedizinisch behandelt" bzw. „es wurde osteopathisch behandelt" ausreichend sei, um die Behandlung von einer "physiotherapeutischen" Behandlung oder "Standardbehandlung" abzugrenzen, wie in manchen Vergleichsstudien formuliert ist. Beispiele hierfür lassen sich zuhauf in einer Übersicht von von Heymann und Klett finden [3]. Deren Aussage im Ergebnis ihrer Analyse führt $\mathrm{zu}$ einem weiteren Sachverhalt, auf den

L. Beyer

Ärztehaus Mitte, Jena, Deutschland

\title{
Manualmedizinisch versus osteopathisch - Zwei Seiten einer Medaille?
}

hier ebenfalls kurz eingegangen werden soll: „Nur wenige Ergebnisse der klinischen Studien oder Metaanalysen ergeben ein positives Bild der osteopathischen $\mathrm{Me}$ dizin, soweit sie über die Manuelle Medizin hinausgeht" [3]. Es wird eine Frage aufgeworfen, die über die Forderung nach Beschreibung der genauen therapeutischen Prozedur hinausgeht, aber eine Antwort noch vermissen lässt: „Wie groß sind eigentlich die Schnittmengen zwischen einer manualmedizinischen und einer osteopathischen Behandlung? bzw. Wie klein sind die Unterschiede zwischen beiden?"

Auch aus anderen Aspekten spielt die promiskuitive Verwendung von osteopathisch und manualmedizinisch keine untergeordnete Rolle. Bis 2014 hatte die Zeitschrift Manuelle Medizin die Untertitel „Chirotherapie und Manuelle Therapie“. Dann wurden die Begriffe im Untertitel erweitert auf: „Chirotherapie, Manuelle Therapie, ärztliche Osteopathie, ärztliche Chiropraktik, muskuloskelettale Medizin“. Insbesondere „ärztliche Osteopathie“ und „ärztliche Chiropraktik“ sollen hier offensichtlich von anderen nichtärztlichen osteopathischen und chiropraktischen Praktiken abgrenzen. Bereits nach einer kurzen Zeit haben die Herausgeber und die Vertreter der manualmedizinischen Gesellschaften Österreichs, der Schweiz und Deutschlands aber diese eher Verwirrung stiftende begriffliche Vielfalt erkannt, die keineswegs, wie beabsichtigt, zu vermehrt wissenschaftlichen Beiträgen in der Zeitschrift beigetragen hat. Inzwischen wurde der Untertitel auf „European Journal of Manual Medicine“ geändert, nicht zuletzt um der euro- päisch-internationalen Entwicklung der manuellen Medizin Rechnung zu tragen [10]. Diese wird vom Zusammenschluss zahlreicher nationaler MM-Gesellschaften in Europa in der ESSOMM (European Society Of Manual Medicine) [4] und in der Organisation wissenschaftlicher Vertreter einer manuellen muskuloskeletalen Medizin (IAMMM - International Academy Manual Musculoskeletal Medicine) [5] geprägt. $\mathrm{Zu}$ den nichtärztlichen Entwicklungen von Chiropraktik und Osteopathie wie auch $\mathrm{zu}$ deren Entwicklung im ärztlichen Bereich haben verschiedene aktuelle Entwicklungen in der Medizin beigetragen, welche eher im kommerziellen Bereich des Gesundheitsmarkts zu suchen sind denn im wissenschaftlichen Bereich. Da eine solche Entwicklung von den unterschiedlichsten Interessen geprägt ist, ist es schwierig, hier oder heute ein Fazit zu ziehen. F. Mildenberger befasste sich im Auftrag der Robert Bosch Stiftung (Institut für Geschichte der Medizin) mit der Geschichte der Chiropraktik, Chirotherapie und manuellen Medizin in Deutschland, die in seinem Buch „Verschobene Wirbel - verschwommene Traditionen" [6] ausführlich mit über 100 (!) Seiten Quellenangabe belegt ist. Am Abschluss des Buches kommt Mildenberger $2015 \mathrm{zu}$ der bemerkenswerten Schlussfolgerung: „Ob aber die Erkenntnisse der Manuellen Medizin wirklich in allen Kliniken und Arztpraxen angekommen sind, bleibt abzuwarten" und "Die unter neoliberalem Einfluss erlassenen Veränderungen auf dem Gesundheitsmarkt nach 2000 gestatten es nun Patienten mit gefüllter Geldbörse und Willen zur Information, 
alle Wirbelsäulentherapien in Anspruch zu nehmen. Die Einsparungen im Gesundheitswesen könnten dazu führen, dass die heute in der medizinischen Angebotsstruktur bestens verankerte Manuelle Medizin sich alsbald subkulturaler Konkurrenzbewegungen erwehren muss" [5].

Als Ausgangspunkt für unsere weiteren Überlegungen, die auch im Herausgebergremium der Zeitschrift $M a-$ nuelle Medizin diskutiert wurden, soll die über viele Jahre gewachsene Definition der MM durch die Deutsche Gesellschaft für MM (DGMM) dienen. Hier heißt es 2015: „Osteopathie ist Bestandteil und Erweiterung der Manuellen Medizin. Sie ist bei vielen von Schmerzen begleiteten Funktionsstörungen und Erkrankungen insbesondere am Bewegungsorgan eine wirksame Alternative bzw. Ergänzung zu medikamentöser Behandlung oder $z u$ operativen Eingrif-

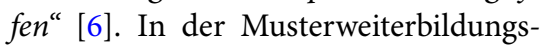
ordnung der Bundesärztekammer von 2019 zur Zusatzweiterbildung MM heißt es: „Die Zusatz-Weiterbildung Manuelle Medizin umfasst in Ergänzung zu einer Facharztkompetenz die Erkennung und Behandlung reversibler Funktionsstörungen des Bewegungssystems einschließlich ihrer Wechselwirkung mit anderen Organsystemen mittels manueller Untersuchungs- und Behandlungstechniken " [7]. Zweisich sehrähnelnde Formulierungen, die eine große Schnittmenge zwischen dem „Manualmedizinischen“ und dem „Osteopathischen“ widerspiegelt. Einen interessanten Hinweis in der Diskussion lieferte Böhni, der auf einem Kongress der SAMM (Schweizerische Ärztegesellschaft für Manuelle Medizin) darauf hinwies, dass der Titel des Lehrbuches von Philip Greenman (Doctor of Osteopathic Medicine, D.O.; Professor an der Michigan State University) „Principles of Manual Medicine"[8] bei der Übersetzung ins Deutsche geändert wurde in „Lehrbuch der Osteopathischen Medizin" [9], obwohl es in Deutschland eine ärztliche Weiterbildung unter diesem Begriff gar nicht gab.

Da es hier aber nicht um gesundheitspolitische Fragen geht, sondern um präzise Darstellungen wissenschaftlicher Publikationen und Erfahrungsberichte aus der Praxis, wurde mehrheitlich vorge- schlagen, den Autoren, wenn sie von einem „osteopathischen Vorgehen“ sprechen, zu empfehlen, besser „manualmedizinisch-osteopathisch“ als Adjektiv zu verwenden. Die Diskussion führte nicht völlig einheitlich zu dieser Meinung. In die Diskussion wurde auch eingebracht, dass der Anspruch, ein European Journal zu sein, auch die Verpflichtung mit sich bringt, die Regelungen in anderen europäischen Staaten anzuerkennen. Da gibt es nicht wenige, die die Osteopathie als Heilberuf eingeführt haben.

Die Problematik der Abtrennung von „nichtärztlichen-Osteopathen“ lässt sich sicher durch die Begutachtung der wissenschaftlichen Qualität der eingereichten Beiträge filtern. Wenn Beiträge mit hoher Qualität abgedruckt werden, sollte davon kein Schaden für die Zeitschrift ausgehen, wenn osteopathisch oder ähnliche Bezeichnungen als Begriffe auftauchen. Allerdings wurde auch schon darauf hingewiesen, dass unterschiedliche Nomenklatur die Kommunikation z.B. zwischen Arzt und Physiotherapeut erschweren können [11].

L. Beyer (federführender Schriftleiter Manuelle Medizin)

\section{Korrespondenzadresse}

\section{Beyer}

Ärztehaus Mitte

Westbahnhofstr. 2, 07745 Jena, Deutschland

lobeyer@t-online.de

Interessenkonflikt. L. Beyer gibt an, dass kein Interessenkonflikt besteht.

\section{Literatur}

1. Vinzelberg S, Alt B, Sachse G et al (2019) Systematisierung und Standardisierung manueller Untersuchungs- und Behandlungstechniken. Man Med 57:341-347. https://doi.org/10.1007/ s00337-019-00605-0

2. Alvarez G, Cerritelli F, Urrutia G (2016) Using the template for intervention description and replication (TIDieR) as a tool for improvingthe design and reporting of manual therapyintervention. Man Ther 24:85-89. https://doi.org/10.1016/j.math. 2016.03.0042

3. von Heymann W, Klett R (2018) Zur Evidenz bei osteopathischen Studien. Man Med 56:374-383. https://doi.org/10.1007/s00337-018-0443-0

4. ESSOMM (2020) https://www.essomm.eu/. Zugegriffen: 13. Apr. 2020

5. IAMMM (2020) http://www.iammm.net/. Zugegriffen: 13. Apr. 2020

6. DGMSM (2015) DGMM Positionspapier zur "Osteopathie" in Deutschland
7. Bundesärztekammer (2019) (Muster-)Weiterbildungsordnung 2018. https://www. bundesaerztekammer.de/fileadmin/user upload/downloads/pdf-Ordner/Weiterbildung/ 20190920_MWBO-2018.pdf. Zugegriffen: 13. Apr. 2020

8. Greenman P (1996) Principles of manual medicine. Lippincott Williams \& Wilkins, Philadelphia

9. Greenman P (2005) Lehrbuch der Osteopathischen Medizin. Haug, Stuttgart

10. Beyer L (2018) European Journal of Manual Medicine. Man Med 56:119-121. https://doi.org/ 10.1007/s00337-018-0399-0

11. Ebert A, Beyer L (2016) Unterschiedliche Nomenklatur erschwert die Kommunikation zwischen Arzt und Physiotherapeut. Man Med 54:322-325. https://doi.org/10.1007/s00337-016-0180-1 